\title{
Research Papers from the 18th International Symposium on Solubility Phenomena and Related Equilibrium Processes (ISSP-18)
}

\author{
Johan Jacquemin $^{1} \cdot$ Magdalena Bendová $^{2} \cdot$ Earle Waghorne $^{3}$
}

Published online: 24 July 2019

(c) Springer Science+Business Media, LLC, part of Springer Nature 2019

The 18th International Symposium on Solubility Phenomena and Related Equilibrium Processes (ISSP-18) was held from 15 to 20 July 2018 in the "Hôtel Oceania l'Univers" in Tours, France. Briefly, ISSP is an established biennial IUPAC symposium organized by the Subcommittee on Solubility and Equilibrium Data gathering international experts on solubility studies or related subjects to exchange new research and concepts.

ISSP-18 in Tours successfully followed a series of previous meetings, the most recent being ISSP-17 (2016, Geneva, Switzerland), ISSP-16 (2014, Karlsruhe, Germany), ISSP15 (2012, Xining, China), ISSP-14 (2010, Leoben, Austria), and ISSP-13 (2008, Dublin, Ireland). The 18th edition of this IUPAC sponsored symposium was organized by the PCM2E Laboratory of the Tours University, with Dr. Johan Jacquemin acting as conference chair. In conjunction with ISSP-18, the 17th annual meeting of the IUPAC Subcommittee on Solubility and Equilibrium Data (SSED) was held on 15 July, chaired by Clara Magalhães.

For 6 days, the ISSP-18 conference brought together over 170 scientists from 34 different countries (Algeria, Australia, Azerbaijan, Botswana, Bulgaria, Canada, China, Czech Republic, Denmark, Finland, France, Germany, Hungary, India, Ireland, Israel, Japan, Mexico, Morocco, Netherlands, Nigeria, Oman, Pakistan, Poland, Portugal, Russia, South Africa, South Korea, Spain, Sweden, Taiwan, Ukraine, United Kingdom, United Sates) with a shared interest in solubility. This symposium addressed the general importance of solubility phenomena and associated physical properties in a variety of settings ranging from green chemistry to nuclear waste disposal and modern technical applications.

Johan Jacquemin

jj@univ-tours.fr

Magdalena Bendová

bendova@icpf.cas.cz

Earle Waghorne

earle.waghorne@ucd.ie

1 Université de Tours, Laboratoire PCM2E, Parc de Grandmont, Avenue Monge, 37200 Tours, France

2 Department of Aerosol Chemistry and Physics, Institute of Chemical Process Fundamentals of the CAS, v. v. i., Rozvojová 135/1, Prague, Czech Republic

3 UCD School of Chemistry, University College Dublin, Dublin, Ireland 
This edition was built on the momentum gained during the previous ISSP meetings with sessions focused on specific topics including data evaluation and consistency, computer assisted modelling, ionic liquids, deep eutectic solvents, molten salts and high ionic strength solutions, solubility and nanoparticles, organics and mixed solvents, to cite few. Furthermore, a Workshop on the "Modelling of Fluid Phase Equilibria and Solubility Phenomena-From Experiments to Their Evaluations" was led by Agilio Padua (École Normale Supérieure de Lyon, France), while a discussion session on specialized topics was organized for the first time in history of this event. This discussion session was a great opportunity to deeply discuss about a proper definition of the so-called "Deep Eutectic Solvents", as well as various hot topics including the use of "Ionic Liquids" for thermal and electronic energy storages.

ISSP-18 invited distinguished plenary and invited lecturers to introduce the main topics. Lecturers were: Donald T. Reed (Repository Science and Operations, Los Alamos National Laboratory, USA), "Solubility of Actinides in Complex Groundwater and Brine Systems"; Marian Paluch (University of Silesia in Katowice, Poland), "Solubility Advantage of Amorphous Drugs"; Margarida F. Costa Gomes (École Normale Supérieure de Lyon, France), "Physical and Chemical Gas Absorption in Ionic Liquid Mixtures"; Agilio Padua (École Normale Supérieure de Lyon, France), "Solubility and Phase Equilibria from Theoretical Chemistry and Molecular Simulation"; Urszula Domanska (Warsaw University of Technology, Poland), "Phase Equilibria in Binary and Ternary Systems from Experiments to their Evaluations"; Patrik Johansson (Chalmers University of Technology, Göteborg, Sweden), "Explicit and Implicit Modelling of Non-Aqueous Electrolytes for Next Generation Batteries"; Richard Buchner (Institute of Physical and Theoretical Chemistry, Universität Regensburg, Germany), "Dielectric Spectroscopy of Ion-Binding Equilibria"; Marijana Blesic (Queen's University Belfast, UK), "Solubility Phenomena in Aqueous Solutions of Zwitterionic Salts"; Ramesh Gardas (Indian Institute of Technology Madras, India), "Phase Equilibrium Studies of Systems Involving Aqueous Ionic Liquids and Deep Eutectic Solvents"; Miroslaw Chorazewski (University of Silesia in Katowice, Poland), "Simple and Accurate Tool for Prediction of Densities and Speeds of Sound of Compressed Liquids using Fluctuation-based Isothermal Equation of State"; Michaël Deschamps (Université d'Orléans, France), "Diffusion in Electrolytes, Inside and Outside Electrodes, as Viewed by Nuclear Magnetic Resonance"; Manuel Maréchal (SyMMES, University of Grenoble Alpes/CNRS/CEA, Grenoble, France), "Soft Electrolytes with Controlled Organisations for Energy Storage and Conversion"; Alexandre Ponrouch (Institut de Ciència de Materials de Barcelona, Spain), "Electrolytes for $\mathrm{Ca}$ and Mg Based Batteries"; Mireille Turmine (UPMC Sorbonne Université, France), "Nanoparticles Solubilities: Nonextensive Thermodynamics Approaches, Applications"; Isabel M. Marrucho (Instituto Superior Técnico, Universidade de Lisboa, Portugal), "Eutectic Solvents: Expanding Chemical Profiles".

This conference included also 58 oral and 22 flash presentations (for young researchers) and a poster session. Poster presentations have been organized in a dedicated poster session, during which 62 posters were greatly presented. Outside the session lecture theatres, numerous exhibitors were also present and all attendees also took the opportunity to deeply improve their knowledge of modelling approaches during our workshop. Thoroughout all presentations, ISSP-18 promoted the participation and exchange of ideas of a broad spectrum of stakeholders-from academics working in science and technology to researchers in chemical, pharmaceutical, medical, materials (to electronic, building, artistic, ... applications) industries-not forgetting the modern environmental concerns (e.g., greenhouse gases solubility, remediation of mining and industrial impacts, mineral recovery from brines and seawater, energy storage applications like metal-ion batteries and 
supercapacitors, etc.), which only can be well predicted and developed if accurate and reliable data are available.

Within ISSP-18, two Franzosini awards (supported by IUPAC-SSED) were presented, given to Maria Gonzalez-Miquel (Universidad Politécnica de Madrid, Spain) and Romain Privat (ENSIC-LRGP, France).

These Franzosini Awards, as well as best presentations Poster and Flash Awards, were celebrated during the Gala dinner at Royal Castle Amboise on Thursday 19 July evening within a typical French fashion!

All together this conference was a great success and we look forward to the next ISSP19 scheduled at the Los Alamos National Laboratory, USA.

This special issue of the Journal of Solution Chemistry contains 16 contributions presented at the symposium. A further 6 papers, based on the invited lectures will be published in an upcoming issue of Pure and Applied Chemistry. These give a flavor of the quality but also of the diversity of the subjects discussed.

Finally, the success of a special issue is not only based on the quality of the authors and their excellent manuscripts but it is an association of joint efforts of the journal's Editorial Team, authors, and of course, reviewers. Again, many thanks for all of your efforts!

Publisher's Note Springer Nature remains neutral with regard to jurisdictional claims in published maps and institutional affiliations. 